\title{
ANÁLISIS DEL RENDIMIENTO TÉRMICO DE UN CALENTADOR SOLAR DE AIRE DE CONVECCIÓN NATURAL CON ABSORBENTE CORRUGADO Y LECHO POROSO
}

\author{
THERMAL PERFORMANCE ANALYSIS OF A NATURAL CONVECTION \\ AIR SOLAR HEATER WITH CORRUGATED ABSORBENT AND \\ POROUS BED
} José Quiñonez-Choquecota ${ }^{{ }^{*}}$ (D)

'Facultad Ingeniería Civil y Arquitectura, Universidad Nacional del Altiplano, Puno, Perú

Recibido (Received): 14 / 11 / 2020 Aceptado (Accepted): 30/ 06 / 2021

\begin{abstract}
RESUMEN
Se analiza el efecto del lecho poroso en un calentador solar de aire con placa absorbente corrugado en $\mathrm{V}$ y un diseño que facilita la libre convección, en términos de parámetros de rendimiento y eficiencia térmica. El colector se evaluó, con y sin lecho poroso, en condiciones climáticas similares al montar sobre una pared vertical. Los resultados revelaron que el flujo másico está directamente afectado con la temperatura ambiente. Se observó que el lecho poroso afecta la subcapa laminar de la placa absorbente generando mayor turbulencia que mejora la tasa de transferencia de calor, por tanto, el colector con lecho poroso presenta mayor temperatura en su interior y disminuye ligeramente su eficiencia. Además, el lecho poroso funciona como almacén de calor que reduce inestabilidades lo cual permite una temperatura de salida uniforme y permite aumentar el tiempo de funcionamiento. El colector es de bajo costo, es respetuoso con el medio ambiente y factible para calefacción de ambientes en zonas frígidas.
\end{abstract}

Palabras Clave: Abastecimiento de energía, balance energético, calefacción solar, transferencia de calor

\section{ABSTRACT}

The effect of the porous bed in a solar air heater with a V-corrugated absorbent plate and a design that facilitates free convection is analyzed, in terms of performance parameters and thermal efficiency. The collector was evaluated, with and without porous bed, under similar climatic conditions when mounted on a vertical wall. The results revealed that the mass flow is directly affected by the ambient temperature. It was observed that the porous bed affects the laminar sublayer of the absorbent plate generating greater turbulence that improves the rate of heat transfer, therefore, the collector with a porous bed presents a higher temperature inside and slightly decreases its efficiency. In addition, the porous bed works as a heat store that reduces instabilities which allows a uniform outlet temperature and allows to increase the operating time. The collector is inexpensive, respectful with the environment and feasible for space heating in frigid areas.

Keywords: energy supply, energy balance, solar heating, heat transfer

\section{INTRODUCCIÓN}

Un colector calentador solar de aire (CSA), es un intercambiador de calor que convierte la energía solar incidente en energía térmica que transfiere al aire que circula por su interior para su aprovechamiento.

Actualmente, las investigaciones de los CSA se han centrado principalmente en mejorar el rendimiento termo hidráulico, reduciendo perdidas

* Corresponding author.:

E-mail: josequch@gmail.com de calor, integrando extensiones en las superficies, alterando la subcapa laminar en las superficies absorbentes e innovando la trayectoria del flujo de aire en los ductos [1-6].

\section{ANTECEDENTES}

La literatura revela que, los CSAs de doble paso en flujo paralelo o contracorriente tienen mayores eficiencias en comparación con un solo paso [7, 8]. Descubrieron también, que la placa absorbente corrugado en $V$ en CSAs de doble paso, aumenta el área de trasferencia de calor mejorando la eficiencia de hasta un $14 \%$ más en comparación con el de placa 
plana [9-11]. Dhiman [8] y Sopian [12] revelaron que un CSA de doble paso integrado el material con un medio poroso, aumenta la tasa de transferencia de calor y su eficiencia es mayor de 10 a $20 \%$ más que el CSA sin material poroso. Todas estas mejoras aumentan notablemente la transferencia de calor y el rendimiento, sin embargo, se presenta mayor caída de presión, lo que demanda más potencia de bombeo del ventilador para lograr mayor eficiencia, limitando su rendimiento térmico en general.

En los CSAs de convección natural, el flujo de aire es inducido por la acción de la fuerza de flotación del aire en lugar de un ventilador, dado que puede aplicar en zonas remotas donde no hay electricidad o para reducir el consumo de la electricidad. Cuzminschi [13] y Hernández [14] examinaron CSAs de doble flujo en paralelo de libre convección con placa absorbente corrugado en $\mathrm{V}$, donde el rendimiento térmico fue de hasta $60,4 \%$. Sin embargo, en la literatura se tienen insuficientes estudios de CSAs de absorbente corrugada en $V$ con lecho poroso, que trabajan por libre convección, que tengan diseños más eficientes con adecuados materiales seleccionados y económicamente viables [15].

Esta investigación tiene como objetivo analizar el efecto del lecho poroso que se integra en un CSA innovador de doble flujo en paralelo, donde el aire circula por convección libre, que tiene como factor de mejora una placa absorbente corrugada en $\mathrm{V}$, una campana en la parte superior del colector que recoge el aire caliente de salida y una forma amplia en la entrada del aire. El nuevo CSA proporciona energía de una forma limpia, sostenible y económica, siendo así, aplicable para solucionar problemas de calefacción de ambientes como en las zonas altoandinas del Perú.

\section{METOdOLOGÍA}

\subsection{DESCRIPCIÓN Y EVALUACIÓN EXPERIMENTAL}

El colector desarrollado tiene dos canales de flujo, el aire recorre los canales en la misma dirección hasta llegar al otro extremo del colector que tiene una forma de campana que vuelve a combinarse de nuevo el aire. Sus dimensiones son 2,29 m de largo, 0,935 m de ancho y $0,126 \mathrm{~m}$ de espesor, la superficie de apertura es $0,835 \mathrm{~m} \times 2.0 \mathrm{~m}$. (figura 1).

Para su construcción se utilizó una placa absorbente de acero inoxidable galvanizado de 0,8 $\mathrm{mm}$ de espesor que fue corrugado en forma de $\mathrm{V}$ con ápice de corrugado de $90^{\circ}$, y se pintó por ambos lados con pintura negro mate resistente a altas temperaturas con una absortancia igual a 0,90. La cubierta trasparente fue de policarbonato alveolar transparente de $6 \mathrm{~mm}$ espesor. La parte interior del colector (Canal 2) fue recubierto con lámina reflectante de aluminio de $0,2 \mathrm{~mm}$ y se utilizó como aislante el poliestireno de $5 \mathrm{~cm}$ de espesor. En la entrada del colector, se tiene una abertura en forma de boquilla de polietileno con área transversal de $2 \mathrm{~cm}$ x $65 \mathrm{~cm}$ y la carcasa fue construida de plancha galvanizado de $0,3 \mathrm{~mm}$ de espesor resistente a climas agresivos. Cabe destacar que los materiales utilizados fueron de bajo costo, que suman en total 450,00 Soles aproximadamente.

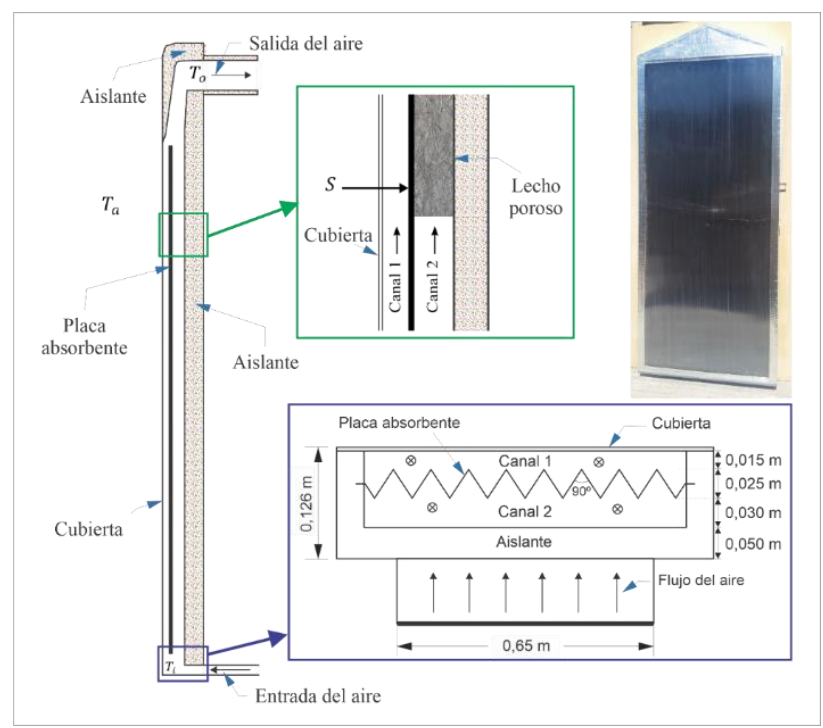

Fig. 1. Esquema lateral y componentes internos del CSA.

La evaluación del colector se realizó exponiéndolo al sol al aire libre en el Centro de Investigación en Energías Renovables CINER, en la región Puno, Perú, (latitud: $-15,823^{\circ}$, longitud: $-70,012^{\circ}$ y 3832 m.s.n.m). Se instaló en una pared vertical con orientación hacia el norte geográfico en una pequeña habitación cumpliendo en parte las pautas establecidas en la norma ASHRAE [16]. El colector se evaluó en dos configuraciones, sin lecho poroso (tipo I) y luego con lecho poroso (tipo II) en condiciones ambientales similares reales para cielo despejado, en los meses de mayo y junio de 2018. Para evaluar el CSA de tipo II, se ha utilizado como lecho poroso la virutilla de acero $\mathrm{N}^{\circ} 4$ de $2 \mathrm{~m}$ de longitud integrándolo en el canal 2. La porosidad del lecho poroso se determinó experimentalmente como, el volumen del agua que llena los resquicios de una muestra porosa entre el volumen que contiene el material poroso y el agua, esta porosidad resultó ser de 96,4\% [17].

Para cada configuración se realizaron cinco ensayos, desde las 8:00 a 16:00 horas, el registro de datos fue realizada con una frecuencia de 10 minutos. Las variables monitoreadas durante los ensayos fueron: las temperaturas de, la placa absorbente, de ambiente, de entrada, de salida del aire, velocidad de aire de salida y radiación solar incidente sobre el colector. Los instrumentos utilizados fueron: dos 
termómetros digitales Fluke modelo 54 IIB con termocupla tipo $\mathrm{K}$, para medir la velocidad del viento se utilizó un anemómetro digital Lutron modelo AM4247SD, la irradiancia de midió con un piranómetro Apogee Intruments modelo MP-240.

\subsection{ANÁLISIS TERMODINÁMICO}

Se desarrollaron modelos matemáticos basados en el balance de energía, que permiten estudiar y calcular la eficiencia de los CSAs, en condiciones estacionarias del flujo de aire y para gradientes de temperaturas solamente en dirección del flujo del aire [18-20]. La eficiencia instantánea es importante para estimar la energía útil que el colector puede producir a condiciones climáticas en un determinado, el cual se calcula mediante:

$$
\eta_{c}=\frac{\dot{m} c_{p}\left(T_{o}-T_{i}\right)}{A_{c} G_{p}}=F_{R} \frac{S}{G_{p}}-F_{R} U_{L} \frac{\left(T_{i}-T_{a}\right)}{G_{p}}
$$

Donde: $A_{c}$ el área de apertura del colector, $S$ es la irradiancia incidente sobre el área de apertura del colector, $\mathrm{T}_{\mathrm{a}}$ es la temperatura ambiente exterior, $\mathrm{T}_{\mathrm{i}} \mathrm{y}$ $\mathrm{T}_{\mathrm{o}}$ son las temperaturas de entrada y salida del aire respectivamente, $G_{p}$ es la irradiancia solar total en el plano inclinado del colector, $F_{R}$ es el factor de extracción del calor, $\mathrm{U}_{\mathrm{L}}$ es el coeficiente global de pérdidas de calor, $\dot{m}$ es el flujo másico total y $c_{p}$ es el calor específico del aire.

Para comparar, los parámetros de transferencia de calor, las características de flujo del aire y la eficiencia térmica entre ambas configuraciones con la teoría desarrollada, se utilizaron, medidas de dispersión, regresión lineal y regresión de superficie de respuesta con Matlab y Minitab. Para analizar la calidad del ajuste entre los valores medidos y los valores simulados para la eficiencia, se determinó la raíz cuadrada del error cuadrático medio RMSE y el valor porcentual de la raíz cuadrada del error cuadrático medio RMSE\%. Estos errores cuantifican la desviación media de los valores medidos mediante la segunda expresión de la ecuación (1) respecto a los valores simulados calculados por la ultima expresión de la ecuación (1).

\section{ANÁLISIS DE RESULTADOS}

\subsection{COMPARACIÓN DE LOS PARÁMETROS DE RENDIMIENTO}

La variación de las temperaturas, de entrada, salida, la placa, ambiente, el flujo másico y la radiación solar en función del tiempo son similares para ambas configuraciones del colector, sin embargo, en la configuración de tipo II se tienen mayores desviaciones debido al cielo claro intermitente que se presentó, (figura 2). Como es evidente, la radiación solar alcanzó su valor máximo al mediodía, razón por la cual la temperatura de salida, del absorbente y del ambiente también lograron las máximas temperaturas al mediodía. El periodo de duración de las temperaturas altas en el colector de tipo II fue mayor en comparación del tipo I. Al comparar las temperaturas promedios máximas y mínimas, y promedios globales, del ambiente, de salida, de entrada y de la placa absorbente, se observó que las condiciones ambientales son similares para ambos casos. En el tipo Il la placa absorbente y el aire de salida, alcanzaron mayores temperaturas máximas promedio que el tipo I.

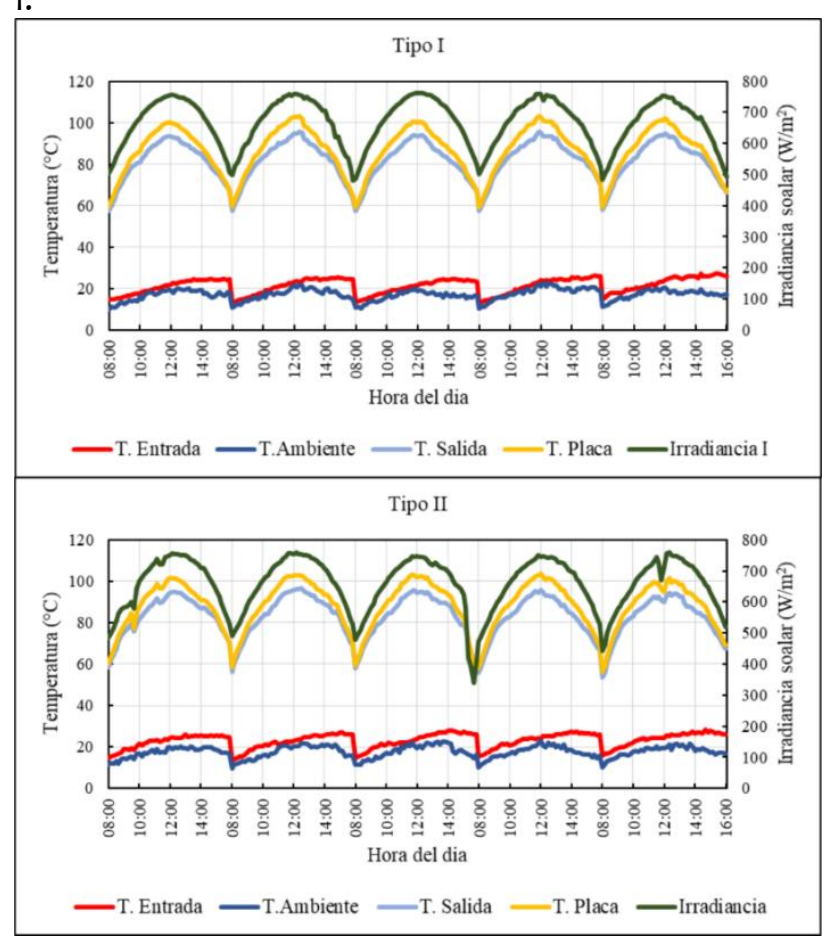

Fig. 2. Variación de los parámetros de rendimiento con el tiempo.

En el lugar de la evaluación, la velocidad del viento recurrente fue de 0 a $1 \mathrm{~m} / \mathrm{s}$ por las mañanas y 2 a $4 \mathrm{~m} / \mathrm{s}$ por las tardes, razón por la cual en la figura 2 se observa una brecha entre la temperatura ambiente y la entrada del aire, esta brecha es más significativa en el tipo II, lo cual significa que existe mayores pérdidas de calor por convección por las tardes. El efecto del viento también afectó al caudal, puesto que la curva de flujo másico no es simétrica alrededor del mediodía como se aprecia en la figura 3. Se observó también, que el flujo másico depende directamente de la velocidad de aire y el comportamiento es similar a la irradiancia solar, es decir a mayor irradiancia solar mayor flujo de masa. Además, se notó que el colector de tipo II tiene una relevante disminución de la velocidad del aire y del flujo másico del $11 \%$ y $9,3 \%$ respectivamente, en comparación al tipo I. 
Ahora bien, una relación interesante que se ha encontrado, es la relación del flujo másico con la brecha existente entre la temperatura de entrada y del ambiente que se presentó por tardes. Para hacer un análisis exhaustivo se realizó un ajuste de los datos involucrados poco comunes para ambas configuraciones, luego, se descubrió, que el flujo másico tiene una relación directa mayor con la temperatura ambiente que con la temperatura de entrada (figura 3).
En la figura todas las anotaciones y letras deben tener como mínimo $2 \mathrm{~mm}$ de altura. Debe evitarse el uso de letras en negrita, ya que se verá oscuro y perdiendo detalles cuando se imprima.

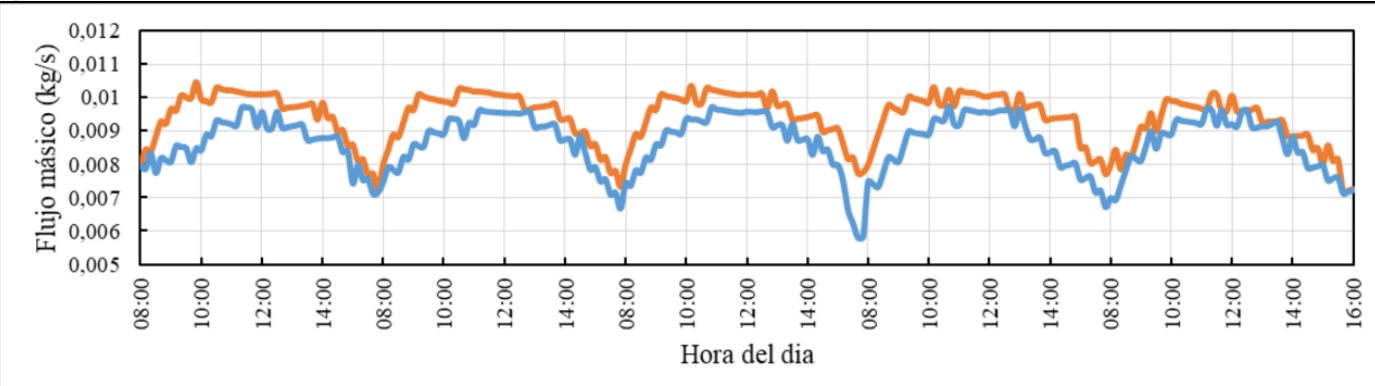

a)

Flujo másico I —Flujo másico II

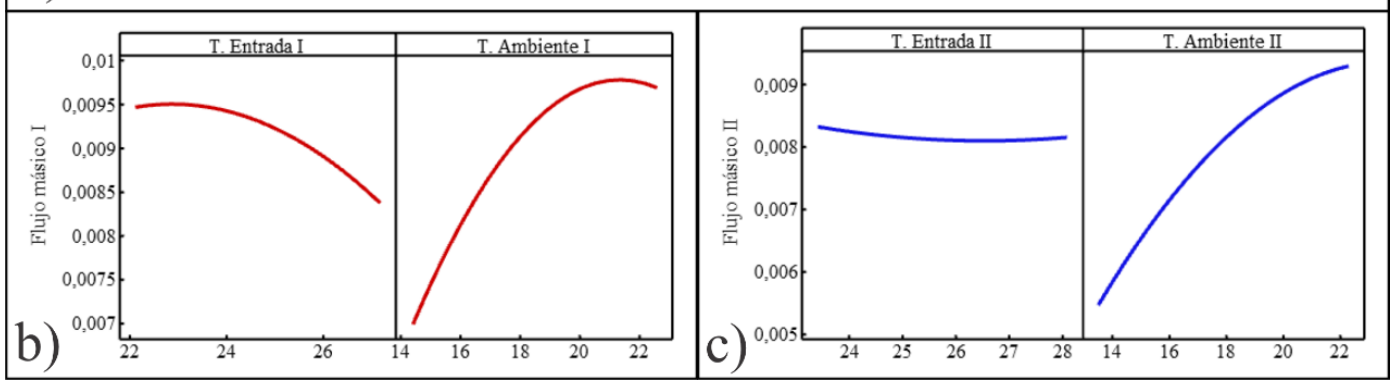

Fig. 3. a) Variación de flujo másico con el tiempo. b) Efectos principales para el flujo másico de tipo l. c) Efectos principales para el flujo másico de tipo II.

\subsection{COMPARACIÓN DE LA EFICIENCIA}

La eficiencia instantánea simulada se obtiene mediante regresión lineal, estas curvas fueron:

$$
\begin{array}{cl}
\text { Tipo I: } & \eta_{c}=0,601-13,346 \frac{T_{i}-T_{a}}{G_{p}} \\
\text { Tipo Il: } & \eta_{c}=0,585-13,003 \frac{T_{i}-T_{a}}{G_{p}}
\end{array}
$$

El coeficiente de determinación de para el tipo I fue 0,80 , mientras que para el tipo II fue de 0,73 , los cuales indican que correlacionan significativamente con los valores medidos. Los factores 0,601 y 0,585 correspondientes a cada configuración y representan la máxima eficiencia, es decir es el rendimiento óptico del colector que sólo depende de las características con las que está fabricado el colector, en este punto el colector de tipo I puede alcanzar mayor eficiencia. Por otro lado, los factores $-13,346$ y $-13,003$ son factores de pérdida de calor correspondientes a cada tipo de configuración, que representa el producto del factor de remoción de calor por el coeficiente total de pérdidas de calor del colector; siendo el ángulo $55^{\circ}$ el ángulo promedio de incidencia, en este punto el que pierde más calor es el tipo II.

Para analizar la calidad del ajuste entre los valores medidos y los valores simulados de la eficiencia, determinamos la raíz cuadrada del error cuadrático lecho RMSE \%. Para el colector tipo I, el RMSE \% fue $6,36 \%$, en cambio para el tipo II fue $7,31 \%$, en consecuencia, las curvas simuladas para las eficiencias (ecuaciones 2 y 3) estiman el rendimiento del colector con un error menor al 8 \% en ambas configuraciones del colector determinado. En cuanto a la eficiencia térmica diaria promedio, para el tipo I fue 51,1\% mientras que para el tipo II fue 47,1\%. Observando la eficiencia medida y simulada del CSA de tipo I es $4 \%$ más eficiente que el tipo II (figura 4).

Por otra parte, se estimaron superficies de respuestas de la eficiencia en función de $x=T_{i}-T_{a} / G_{p}$ y la Irradiancia para ambas configuraciones, exceptuando los valores poco comunes mediante Matlab y Minitab. Las superficies de respuesta estimadas fueron obtenidas con un coeficiente de determinación de $92,68 \%$ y $81,04 \%$ para las 
configuraciones de tipo I y tipo II respectivamente, y las desviaciones estándares fueron menores al $6 \%$, lo que indica su importante significado. Con ellas se comprobaron la dependencia lineal de la eficiencia y el factor $x$ mediante el diagrama de Pareto y grafica de los efectos principales.

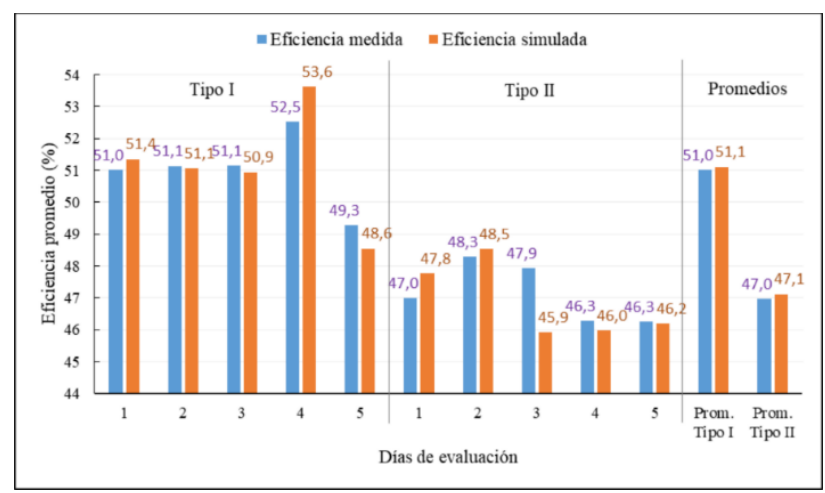

Fig. 4. Comparación de la eficiencia promedio medida y simulada.

Observando los contornos de las dos superficies de respuesta (figura 5), el colector de tipo I es más probable que alcance mayor eficiencia a diferencia del colector de tipo II y el valor óptimo de la eficiencia térmica fue de $61,2 \%$ para una radiación de $762 \mathrm{~W} / \mathrm{m} 2$ en el tipo I a diferencia del 57,1 \% para una radiación de $759 \mathrm{~W} / \mathrm{m} 2$ en el tipo II. Otro resultado revelador es que el lecho poroso permite una temperatura de salida más uniforme, este efecto se refleja en las superficies de respuestas estimadas, donde se observa que el tipo II supera al tipo I para baja radiación.

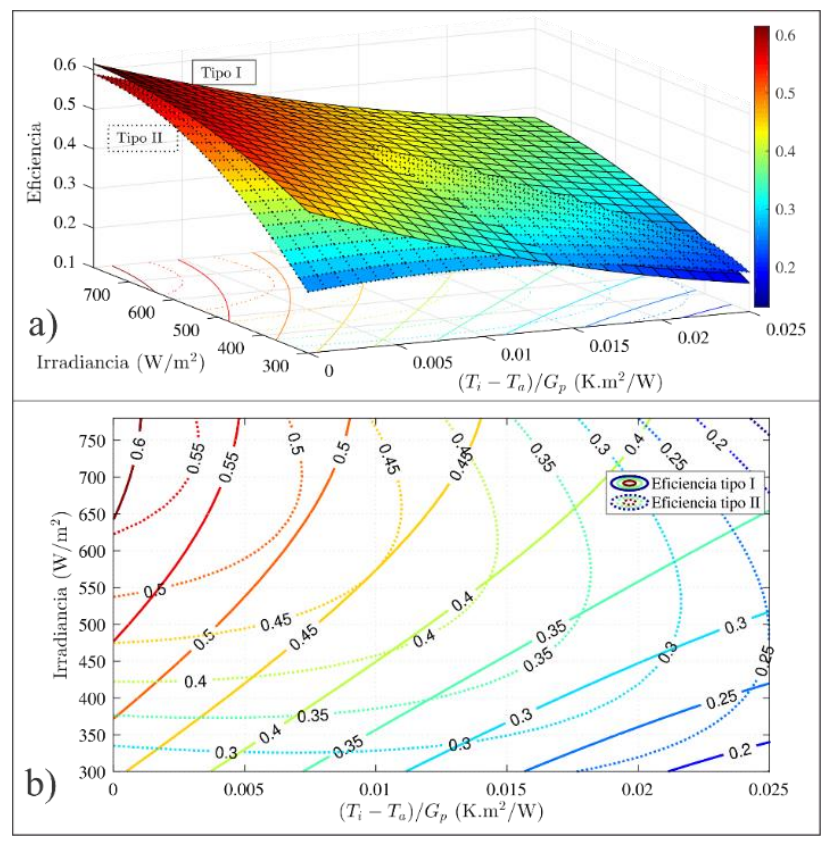

Fig. 5. a) Superficie de respuesta estimada del (SA. b) Contorno de la superficie de respuesta.
El novedoso CSA transfiere mejor el calor mediante el absorbente corrugado en $\mathrm{V}$, puesto que aumenta la superficie de intercambio del absorbente con el aire que circula, también el colector tiene una altura óptima de los canales de tal manera que tiene una geometría y apropiada combinación de sus componentes [11, 21]. El colector de tipo I tiene similar comportamiento que el ejemplar dé Hernández [14] a diferencia de que el nuestro es de mayor eficiencia y mayor temperatura de salida que atribuye a su forma novedosa, sin embargo, el flujo másico es menor. El prototipo de Cuzminschi [13] es de mayor eficiencia, pero en el nuestros la temperatura de salida y la velocidad de salida del aire son mayores. Por consiguiente, colector se puede considerar de alta eficiencia de su clase en ambas configuraciones a pesar de la desventaja de la densidad del aire por la altura sobre el nivel del mar [22].

Un revelador hallazgo es que el flujo másico tiene una mayor relación directa con la temperatura ambiente que con la temperatura de entrada, éste resultado está en la misma línea con lo revelado por Kumar [23], lo cual refuerza la percepción de tener mayor cuidado en el diseño de la cubierta.

Al incorporar el material poroso al colector, se altera la subcapa laminar sobre la placa absorbente generándose efectos de turbulencia y aumenta la tasa de transferencia de calor por la alta conductividad térmica del lecho porosos, induciendo a una distribución más uniforme de la temperatura de salida en comparación con la obtenida para el CSA sin el material poroso, ya que el lecho poroso funciona como almacén de calor que reduce inestabilidades que es importante sobre todo para para días parcialmente nublados y aumentar el tiempo de suministro de calor en un rango estable [24]. La eficiencia térmica ha sido afectada ligeramente al integrarse el material poroso por ser de libre convección, pero la temperatura de salida de aire es mayor. Por otro lado, el estudio ha sido limitado a convección natural del aire, ya que si el aire circulase por convección forzada la eficiencia térmica aumentaría, pero también aumentaría la caída de presión como señalan Rashidi [25] y Jouybari [26], que puede ser estudiado a futuro.

El CSA está diseñado para ser montado en una pared vertical de invernaderos u otros ambientes donde exista necesidad de calefacción como ocurre en las regiones altoandinas del Perú, asimismo es energía limpia, económicamente viable por tener un bajo costo inicial y no requiere una energía adicional para su funcionamiento. 


\section{CONCLUSIONES}

- El calentador solar de aire, tiene un diseño óptimo, el absorbente corrugado en $\mathrm{V}$ aumenta el área de trasferencia de calor y el material poroso aumenta la turbulencia. La eficiencia promedio simulada es $51,1 \%$ y $47,1 \%$ para el tipo I y II respectivamente, con un RMSE\% menor al $8 \%$. El colector sin lecho poroso es ligeramente más eficiente que el sin lecho poroso, sin embargo, ambos colectores tienen un buen rendimiento en comparación con la literatura. El flujo másico es afectado mayormente en forma directa con la temperatura ambiente que, con la temperatura de entrada del aire, dicho efecto permite mayor pérdida de calor al ambiente por las tardes.

- El efecto del lecho poroso al integrar al colector, es alterar la subcapa laminar de la placa absorbente generándose mayor turbulencia que mejora la tasa de transferencia de calor, por consiguiente, eleva la temperatura en su interior del colector y disminuye ligeramente la eficiencia en comparación con el colector sin lecho poroso. El lecho poroso interviene como almacén de energía térmica que permite una temperatura de salida uniforme a pesar de su alta porosidad, lo cual es ventajoso en días parcialmente nublados y para prologar el tiempo de uso. El colector proporciona energía de una forma sostenible, amigable con el medio ambiente, es económicamente viable por su bajo costo inicial y está diseñada para solucionar problemas de calefacción en regiones altoandinas.

\section{REFERENCIAS}

[1] S. Singh y P. Dhiman, "Thermal performance of double pass packed bed solar air heaters - A comprehensive review," Renewable and Sustainable Energy Reviews, vol. 53, pp. 1010-1031, 2016. doi: https://doi.org/10.1016/j.rser.2015.09.058.

[2] S. Chamoli, R. Chauhan, N. S. Thakur, y J. S. Saini, "A review of the performance of double pass solar air heater," Renewable and Sustainable Energy Reviews, vol. 16, no. 1, pp. 481-492, 2012. doi: https://doi.org/10.1016/j.rser.2011.08.012.

[3] F. K. Forson, M. A. A. Nazha, y H. Rajakaruna, "Experimental and simulation studies on a single pass, double duct solar air heater," Energy Conversion and Management, vol. 44, no. 8, pp. 1209-1227, 2003. doi: https://doi.org/10.1016/S0196-8904(02)00139-5

[4] P. T. Saravanakumar, D. Somasundaram, y M. M. Matheswaran, "Thermal and thermo-hydraulic analysis of arc shaped rib roughened solar air heater integrated with fins and baffles," Solar Energy, vol. 180, pp. 360-371, 2019. doi: https://doi.org/10.1016/j.solener.2019.01.036

[5] S. Singh, "Experimental and numerical investigations of a single and double pass porous serpentine wavy wiremesh packed bed solar air heater," Renewable Energy, vol. 145 pp. 1361-1387, 2020. doi: https://doi.org/10.1016/j.renene.2019.06.137

[6] I. Singh y S. Singh, "A review of artificial roughness geometries employed in solar air heaters," Renewable and Sustainable Energy Reviews, vol. 92, pp. 405-425, 2018. doi: https://doi.org/10.1016/j.rser.2018.04.108

[7] T. Alam y M.-H. Kim, "Performance improvement of double-pass solar air heater - A state of art of review," Renewable and Sustainable
Energy Reviews, vol. 79, pp. 779-793, 2017. doi: https://doi.org/10.1016/j.rser.2017.05.087

[8] P. Dhiman, N. S. Thakur, A. Kumar, y S. Singh, "An analytical model to predict the thermal performance of a novel parallel flow packed bed solar air heater," Applied Energy, vol. 88, pp. 2157-2167, 2011. doi: https://doi.org/10.1016/j.apenergy.2010.12.033

[9] A. A. El-Sebaii, S. Aboul-Enein, M. R. I. Ramadan, S. M. Shalaby, y B. M. Moharram, "Investigation of thermal performance of-double pass-flat and v-corrugated plate solar air heaters," Energy, vol. 36, no. 2, pp. 1076-1086, $2011 . \quad$ doi: https://doi.org/10.1016/j.energy.2010.11.042

[10] M. Hedayatizadeh, F. Sarhaddi, A. Safavinejad, F. Ranjbar, y H. Chaji, "Exergy loss-based efficiency optimization of a doublepass/glazed v-corrugated plate solar air heater," Energy, vol. 94, pp. 799-810, 2016. doi: https://doi.org/10.1016/j.energy.2015.11.046

[11] M. A. Karim y M. N. A. Hawlader, "Performance investigation of flat plate, v-corrugated and finned air collectors," Energy, vol. 31 no. 4, pp. 452-470, 2006. doi: https://doi.org/10.1016/j.energy.2005.03.007

[12] K. Sopian, M. A. Alghoul, E. M. Alfegi, M. Y. Sulaiman, y E. A. Musa, "Evaluation of thermal efficiency of double-pass solar collector with porous-nonporous media," Renewable Energy, vol. 34, no. 3, pp. 640-645, 2009. doi: https://doi.org/10.1016/j.renene.2008.05.027

[13] M. Cuzminschi, R. Gherasim, V. Girleanu, A. Zubarev, y I. Stamatin, "Innovative thermo-solar air heater," Energy and Buildings, vol. 158, no. 1, pp. 964-970, 2018. doi: https://doi.org/10.1016/j.enbuild.2017.10.082

[14] A. L. Hernández y J. E. Quiñonez, "Experimental validation of an analytical model for performance estimation of natural convection solar air heating collectors," Renewable Energy, vol. 117, pp. 202-216, 2018. doi: https://doi.org/10.1016/j.renene.2017.09.082

[15] A. P. Singh, A. Kumar, Akshayveer, y O. P. Singh, "Natural convection solar air heater: Bell-mouth integrated converging channel for high flow applications," Building and Environment, vol. 187, 2021. doi: https://doi.org/10.1016/j.buildenv.2020.107367

[16] ASHRAE-Standard, "Methods of Testing to Determine the Thermal Performance of Sollar Collectors," American Socienty of Heating, Refrigeration, and Air Conditioning Engineers, Atlanta 932003.

[17] P. Naphon, "Effect of porous media on the performance of the double-pass flat plate solar air heater," International Communications in Heat and Mass Transfer, vol. 32, no. 1-2, pp. 140-150, 2005. doi: https://doi.org/10.1016/j.icheatmasstransfer.2004.11.001

[18] J. A. Duffie y W. A. Beckman, Solar Engineering of Thermal Processes, 4 ed. John Wiley \& Sons, Ltd, 2013.

[19] K. S. Ong, "Thermal performance of solar air heaters: Mathematical model and solution procedure," Solar Energy, vol. 55, no. 2, pp. 93-109, 1995 . doi: https://doi.org/10.1016/0038$092 X(95) 00021-1$

[20] A. L. Hernández y J. E. Quiñonez, "Analytical models of thermal performance of solar air heaters of double-parallel flow and doublepass counter flow," Renewable Energy, vol. 55, pp. 380-391, 2013. doi: https://doi.org/10.1016/j.renene.2012.12.050

[21] S. N. Saha y S. P. Sharma, "Performance Evaluation of Corrugated Absorber Double Flow Solar Air Heater Based on Energy, Effective and Exergy Efficiencies," International Journal of Mechanical \& Mechatronics Engineering, vol. 17, no. 1, pp. 63-76, 2018.

[22] J. Quiñonez Choquecota, "Investigación experimental de un calentador solar de aire de doble flujo de convección natural de alta eficiencia," Journal of High Andean Research, vol. 21, no. 4, pp. 274282, 2019. doi: http://dx.doi.org/10.18271/ria.2019.504

[23] D. Kumar y B. Premachandran, "Effect of atmospheric wind on natural convection based solar air heaters," International Journal of Thermal Sciences, vol. 138, pp. 263-275, 2019. doi: https://doi.org/10.1016/j.ijthermalsci.2018.12.010

[24] S. Vijayan, T. V. Arjunan, A. Kumar, y M. M. Matheswaran, "Experimental and thermal performance investigations on sensible storage based solar air heater," Journal of Energy Storage, vol. 31, 2020. doi: https://doi.org/10.1016/j.est.2020.101620

[25] S. Rashidi, J. A. Esfahania, y A. Rashidi, "A review on the applications of porous materials in solar energy systems," Renewable 
and Sustainable Energy Reviews, vol. 73, pp. 1198-1210, 2017. doi: https://doi.org/10.1016/j.rser.2017.02.028

[26] N. F. Jouybari y T. S. Lundström, "Performance improvement of a solar air heater by covering the absorber plate with a thin porous material," Energy, vol. 190, p. 116437, 2020. doi: https://doi.org/10.1016/i.energy.2019.116437

Los artículos publicados por TECNIA pueden ser compartidos a través de la licencia Creative Commons: CC BY 4.0. Permisos lejos de este alcance pueden ser consultados a través del correo revistas@uni.edu.pe 